\title{
Interactive Web Maps: Usability Heuristics Proposal
}

\author{
Juliana Orro Marquez $^{\mathrm{a} *}$, Paulo Meirelles ${ }^{\mathrm{b}}$, Tiago Silva da Silva ${ }^{\mathrm{a}}$ \\ ${ }^{a}$ Institute of Technology, Federal University of São Paulo, Brazil, \{juliana.marquez,silva.tiago\}@unifesp.br \\ ${ }^{b}$ Center of Mathematics, Computation, and Cognition, Federal University of ABC,Brazil, paulo.meirelles@ufabc.edu.br \\ * Corresponding Author
}

\begin{abstract}
With the evolution of technology, maps have changed how they are produced and consumed. In the 1990s, along with the internet uprise, printed and digital maps began to be shared and viewed on the web, which provided more significant user interaction with the map and geographic data. However, the ease of creating interactive maps using computational resources sometimes neglects cartographic concepts, impairing the interpretation of geographic data and the quality of the interaction between user and system. This work presents ten specific Usability Heuristics for Interactive Web Maps to identify and elaborate a set of criteria that help create and evaluate the quality of interactive web maps. For this, we used a methodology to develop domain-specific Usability Heuristics, composed of eight steps. This paper presents the ten heuristics elaborated along with the attributes of the name, ID, category and definition, and an additional checklist. This new set encompasses both the concepts of cartography and usability, contributing to better user interaction with the system and geographic data.
\end{abstract}

Keywords: Usability Heuristics, Interactive Web Maps, Human-Computer Interaction

\section{Introduction}

With the evolution of technology, cartography has undergone significant advances provided by technological innovations, the spread of the internet, and electronic devices with integrated geolocation systems. These changes have transformed the way maps are produced, stored, and displayed. Traditional cartography, previously disseminated only on physical media, is now produced in digital media and made available through the internet as digital maps, enabling greater user interactivity with geographic information.

Geographic visualization systems through a computational interface made the representation and analysis of complex data possible. However, the ease of creating interactive maps using computational resources sometimes neglects cartographic concepts, impairing the interpretation of geographic data and the quality of interaction. Faced with this problem, recent studies in cartography reported the need to create guidelines for application in geographic visualization systems (Vincent et al., 2019, Roth et al., 2017). Moreover, there is a lack of methods and techniques in cartographic research to assess usability in user-map interaction (Griffin et al., 2017a).

In this scenario, we are working with quality criteria of the usage and existing evaluation methods in Human-Computer Interaction (HCI). We aim to cover the gap presented in the literature and propose a guide to develop and evaluate the cartographic interface, focusing on usability heuristics, commonly used for evaluating system heuristics.

In the context of the interface, the ten usability heuristics of the Nielsen and Molich (1990) are the most widespread in the literature. However, these heuristics cover interface analysis generally without considering application specifications. Thus, authors have created new usability heuristics to identify problems in domain-specific applications (Kuparinen et al., 2013, Rusu et al., 2011, Solano et al., 2011).

This work aims to improve the interaction between users and the geographic visualization system by creating usability heuristics for interactive web maps. So that developers, designers, and experts use these specific heuristics to design and evaluate systems, ensuring that cartographic concepts and usability attributes are met. For this, we use the methodology for the development of domain-specific heuristics proposed by Quiñones et al. (2018). This approach presents a concrete and robust method, consisting of eight steps that range from gathering information relevant to the topic to validating the proposed heuristics. Also, it has the advantage of having non-linear steps to add new information, creating new iterations during the process.

As a result, we propose ten heuristics with their attributes: name, ID, category and definition, and an additional checklist. This new set of heuristics encompasses both the concepts of cartography and usability, contributing to better user interaction with the system and geographic data. The proposed heuristics help developers of interactive maps on the web to create systems that provide the user with quality interaction, ensuring cartographic and usability concepts. Furthermore, they can also be used as a reference for developing future usability heuristics in other domains of cartography.

This paper is organized as follows: Section 2 presents a 
theoretical framework. Section 3 presents the related works Section 4 describes the methodology used to develop the heuristics. Section 5 details the outputs obtained in each step. Section 6 presents the usability heuristics proposed for interactive maps on the web. Section 7 presents the validation and refinement steps to be performed, and Section 8 presents the conclusions.

\section{Background}

Usability is a quality criterion used in HCI to evaluate the user interaction with a system. Through this quality analysis, it is possible to measure how much the user can achieve their goals in a given context and application (ISO, 1998). Nielsen and Molich (1990) relate usability with the ease of learning and using an interface and the satisfaction generated as a result of the system operation. Thus, we have usability as a quality criterion used to assess the usability of an interface, considering the interaction between user and system applied to a context of use.

In HCI, different evaluation methods can be employed to carry out the analysis of the system and verify its usability. One of them is the Heuristic Evaluation, an inspection method that analyzes a product or interface to identify usage problems. This method is fast, cheap, and practical to verify and predict possible failures in the user-system interaction.

Nielsen and Molich (1990) introduced the term "usability heuristics". It can be understood as a guide composed of essential elements for the composition of an interface and, consequently, for the user's interaction with the system. These sets help experts to cover system specifications, identify and fix problems.

\section{Related Work}

We analyzed works in the areas of web cartography and interactive maps. About guidelines for interactive maps, Tolochko (2016) describes the map elements that undergo modifications in the conversion from physical to digital, adapted from Muehlenhaus (2013). Based on a survey carried out with professionals in cartography who work in the production of interactive maps on the web, the author presents the results identified for the analysis of the workflow, best practices, and evaluation of interactive maps practiced by these professionals. These data are essential elements to understand the process of developing these maps in practice.

We sought papers that presented heuristics or guidelines applied to other areas related to web cartography. Nivala et al. (2008) present an evaluation carried out in four web mapping sites, seeking to identify what the possible existing usability problems would be. The authors identified 403 usability issues related to the user interface, visualization, and map tools. Therefore, the proposed design guidelines for web mapping sites. However, the development of these metrics was not based on a specific methodology for creating guidelines, and they did not go through a validation process, different from the work presented here.
About GeoWeb and GIS Web, studies identified usability problems in these kinds of systems. Komarkova et al. (2007) report the performance of a heuristic evaluation of a website in which they used 138 heuristics, developed by them throughout the study, divided into nine categories. Komarkova et al. (2011) discuss the advantages and disadvantages of heuristic evaluation methods, and the set is composed of 92 heuristics divided into five groups. After a detailed analysis, we used only the classifications and descriptions referring to the formulated groups as elements from that work since they present information relevant to the composition of the usability heuristics.

Kuparinen et al. (2013) conducted a study that deals with usability heuristics for mobile map applications. The authors describe ten specific usability heuristics developed from a theoretical-conceptual approach applied to Nielsen's heuristics. Unlike other works, Kuparinen et al. (2013) validated the heuristics by applying tests with experts using both the proposed heuristics and Nielsen's heuristics. Despite the difference in the application of heuristics, this approach is similar with we used to validate the heuristics in this work.

Outside the scope of web cartography and interactive maps, we analyzed works that could aggregate content with metrics linked to User-Centered Design (UCD). Victorelli and Reis (2020) present guidelines for design on Human-Data Interaction with a focus on information visualization. Although not validated, their results help us understand and how to elaborate usability heuristics, mainly related to the user interface.

Other studies are related to implementing the methodology for developing domain-specific heuristics. Vi et al. (2019) present 11 user experience guidelines for designing extended reality applications. The presented elements were not applied directly in creating the heuristics. However, the content presented when implementing the methodology was relevant for understanding and elaborating the heuristics in this work.

\section{Research Method}

We apply the methodology proposed by Quiñones et al. (2018) to develop domain-specific usability heuristics for interactive web maps. Their methodology comprises eight steps: exploratory, experimental, descriptive, correlational, selection, specification, validation, and refinement. With this framework, the methodology allows formulation, specification, and validation for new sets of heuristics and iterations during the process. Below, we present the methodological steps and their inputs and outputs.

(Step 1) Exploratory: The researcher must collect information about the specific domain being worked on, such as general and specific characteristics, types or classifications, applications, and target audience.

- Input: domain of the specific application that needs a new set of heuristics. 
- Output:

(0) Application information.

(1) Usability attributes.

(2) Set of heuristics and/or other relevant elements.

(Step 2) Experimental: This step is optional. Information are obtained from studies or experiments carried out by external authors.

- Input: domain of the specific application that needs a new set of heuristics.

- Output:
(3) Additional application-specific features.
(4) Usability issues detected.
(5) Problems with existing heuristics.

(Step 3) Descriptive: The information acquired must be sorted and prioritized according to its degree of relevance. Values are assigned based on a score: 3 (very important), 2 ( not very important), 1 (not important).

- Input:(0), (1), (2), (3), (4).

- Output:

(6) Selected information about the application.

(7) Resources selected from the specific domain.

(8) Selected usability attributes.

(9) Set of heuristics and/or other relevant elements.

(Step 4) Correlational: The researcher must associate the information classified in descriptive step with usability heuristics or other relevant elements.

- Input:(6), (7), (8), (9).

- Output:

(10) Corresponding features, attributes, and existing heuristics.

(11) Categories.

(Step 5) Selection: The selected information is analyzed again, and actions are applied to maintain, adapt, create and/or eliminate the content of this information.

- Input:(9), (10).

- Output:

(12) Classified heuristics.

(Step 6) Specification: The number of heuristics and their attributes are defined.

- Input:(5), (10), (11), (12).

- Output:

(13) Set of proposed heuristics.

(Step 7) Validation: The created heuristics go through the validation process to authenticate the proposed set.

- Input:(13).

- Output:

(14) Results of the Heuristic Evaluation.

(15) Results of expert opinion.

(16) Test results with users.
(Step 8) Refinement: The set of heuristics is refined according to the results of the validation step and new usability heuristics for the domain are presented.

- Input:(14), (15), (16).

- Output:

(17) Refining document.

\section{Results}

The methodology presented by Quiñones et al. (2018) is composed of eight steps, of which five steps were performed (exploratory, descriptive, correlational, selection, and specification); the results obtained in each step performed are presented below:

In the Exploratory stage, elements were identified that presented information on: interactive web maps (Table 1, 2, 3 ), heuristic or usability attributes (Table 4, 5, 6) e heuristics or domain-specific relevant elements (Table 7).

The tables present the elements referring to the Exploratory stage and their values in the Descriptive stage. We evaluated the information on a scale from 1 to 3 , according to their importance for constructing the heuristics. We apply the value criterion 1 for non-relevant ones, 2 for those relevant to the elements, but not the creation of heuristics, and 3 are essential for creating the set of usability heuristics.

\begin{tabular}{|l|c|}
\hline Element & Value \\
\hline Interactive map (Roth (2013)) & 3 \\
Web Cartography (Shekhar et al. (2017)) & 3 \\
Web mapping (Shekhar et al. (2017)) & 3 \\
Web GIS (Shekhar et al. (2017)) & 2 \\
Geovisual Analysis (Robinson et al. (2017)) & 3 \\
Interactivity (Roth et al. (2017)) & 3 \\
\hline
\end{tabular}

Table 1. Domain-specific definitions

Table 1, output (0) and (7), presents the definitions referring to the specific domain of interactive maps on the web and cartographic elements with web applications. Among the concepts obtained with these definitions, only the term Web GIS was classified as 2. Despite being related to web cartography, Web GIS is geared towards data processing; that is, the manipulation and interaction of spatial data occur differently from interactive maps.

\begin{tabular}{|l|c|}
\hline Element & Value \\
\hline Static & 2 \\
Dynamic-animated & 2 \\
Dynamic-Interactive & 3 \\
\hline
\end{tabular}

Table 2. Types of classifications

Table 2, output (0) and (7), contains information about types or classifications. Interactive maps have three classifications: static, dynamic-animated, and dynamic-interactive (Sack, 2017). In this case, we classified the first two elements (Static and Dynamic-animated) as 2, related to the content. However, only the last one (Dynamic-Interactive) received 3 as value for presenting the description of the discussed elements. 


\begin{tabular}{|l|c|}
\hline Element & Value \\
\hline Graphic primitives (White (2017)) & 3 \\
Visual variables (White (2017)) & 3 \\
Geovisualization (Dykes et al. (2005)) & 3 \\
Interactive (Roth et al. (2017)) & 3 \\
Maps components (Griffin et al. (2017b)) & 3 \\
\hline
\end{tabular}

Table 3. Domain-specific features

In Table 3, about specific characteristics of the domain, all elements presented received 3 as value, as they have essential information for the creation of heuristics, referring to output (0) and (7).

\begin{tabular}{|l|c|}
\hline Attribute & Value \\
\hline Efficiency & 1 \\
Effectiveness & 1 \\
Satisfaction & 1 \\
\hline
\end{tabular}

Table 4. Attribute of ISO 9241-11

\begin{tabular}{|l|c|}
\hline Attribute & Value \\
\hline Learnability & 3 \\
Efficiency & 3 \\
Memorability & 3 \\
Errors & 3 \\
Satisfaction & 3 \\
\hline
\end{tabular}

Table 5. Nielsen's attributes

The attributes from ISO 92141-11, referring to Table 4, received the value 1 for having elements that are also presented by Nielsen's attribute in Table 5. To eliminate redundancy, we chose to discard it by classifying all the attributes in Table 5 with 3 as value. The result refers to the output (1) and (8).

In Table 6, output (1) and (8), all heuristics presented received the value 3, since, in this work, we use Nielsen's heuristics as control heuristics. For this reason, all were classified as very important.

Table 7, output (2) and (9), refers to sets of heuristics and other relevant elements related to interactive maps on the web. In this table, only the set of elements referring to Geospatial Big data was discarded, as, despite having a relationship with spatial data, it did not present relevant information for the creation of the heuristics.

In the Correlational step (Figure 1), we analyzed the information and correlated them with Nielsen's Heuristics in two distinct analyzes (output 10). A usability expert conducted the first analysis, and a cartographer executed the second. We grouped the information according to the correlation made. The correlations that received a different association from the two specialists were analyzed again until identifying the best correlation. We grouped the data at the end of this step, resulting in seven categories (output 11): user interface, map, interactivity, search operations, responsive design, metadata, and data output.

In the Selection stage (Figure 2), output (12), we analyzed the grouped information within each category. We carried

\begin{tabular}{|l|c|}
\hline Nielsen's Usability Heuristic & Value \\
\hline N1: Visibility of system status & 3 \\
N2: Match between system and the real world & 3 \\
N3: User control and freedom & 3 \\
N4: Consistency and standards & 3 \\
N5: Error prevention & 3 \\
N6: Recognition rather than recall & 3 \\
N7: Flexibility and efficiency of use & 3 \\
N8: Aesthetic and minimalist design & 3 \\
N9: Help users recognize, diagnose, and re- & 3 \\
cover from error & 3 \\
N10: Help and documentation & \\
\hline
\end{tabular}

Table 6. Nielsen's usability heuristic

\begin{tabular}{|l|c|}
\hline Element & Value \\
\hline Geospatial Big data (Robinson et al. (2017)) & 1 \\
\hline interactive web map design (Tolochko (2016)) & 3 \\
\hline Interactive maps (Roth (2013)) & 3 \\
\hline web mapping sites (Nivala et al. (2008)) & 3 \\
\hline Geo Web (Komarkova et al. (2007)) & 3 \\
\hline Web GIS (Komarkova et al. (2011)) & 3 \\
\hline $\begin{array}{l}\text { Human-data interaction (Victorelli and Reis } \\
\text { (2020)) }\end{array}$ & 3 \\
\hline $\begin{array}{l}\text { Mobile Map Applications (Kuparinen et al. } \\
(2013) \text { ) }\end{array}$ & 3 \\
\hline
\end{tabular}

Table 7. Características específicas do domínio

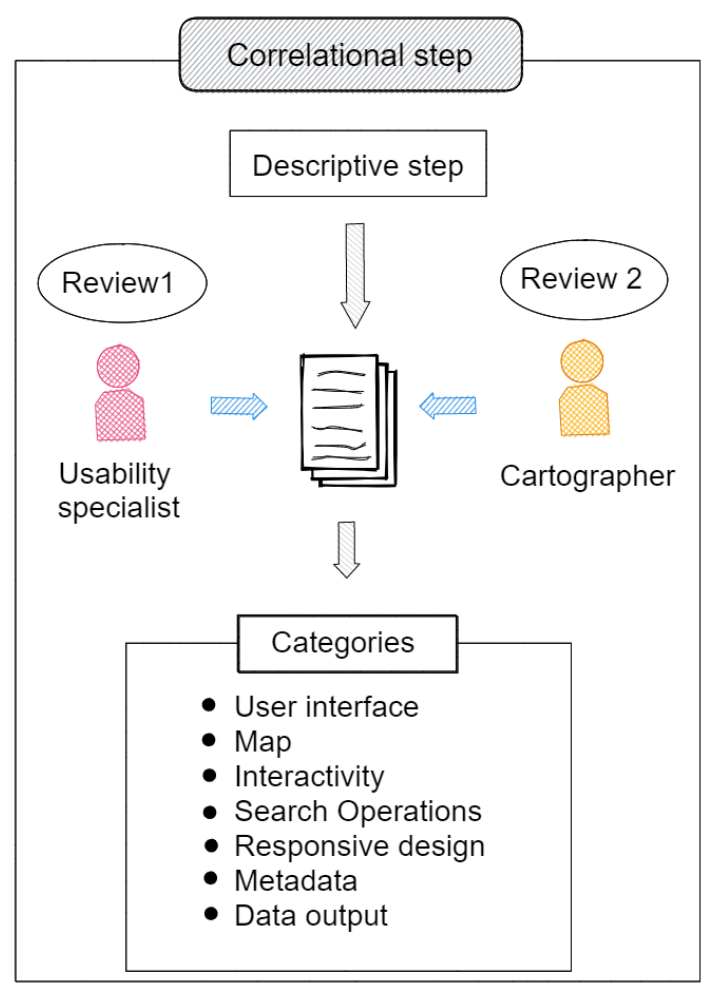

Figure 1. Representation of correlational step.

out actions to maintain, adapt or eliminate information to exclude the ambiguous ideas. After performing these actions, we finished this step with ten categories: user interface, map visualization, scale, symbology, interactivity, research, responsive design, metadata, complementary in- 


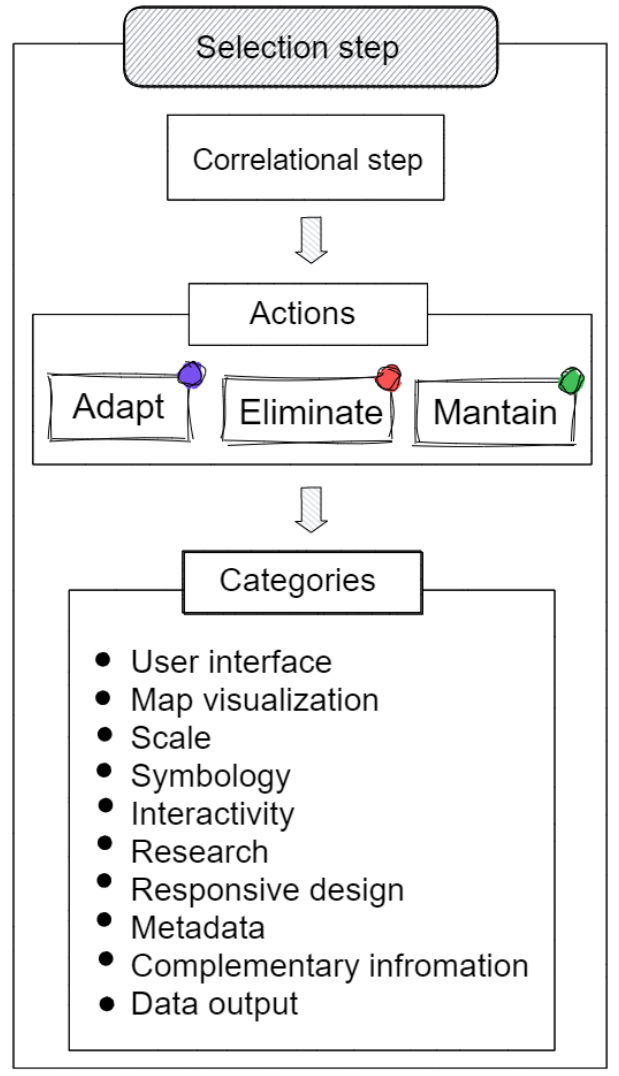

Figure 2. Representation of selection step.

formation, and data output.

Finally, in the Specification step (Figure 3), we added the attributes (ID, name, and definition) for each created category in the Selection step, and all its information became part of the "additional checklist" of the category, referring to output (13). The result of the Usability Heuristics is shown in Section 6.

\section{Usability Heuristics of Interactive Web Map}

\subsection{Agreement and user-friendliness of the interface \\ ID: M1}

Category: User interface

Definition: The application should be easy to use, functional, and intuitive for the user at first glance. Prevent the user from getting lost, minimize information overload, and organize data and tools simply and intuitively (Komarkova et al., 2011, 2007, Kuparinen et al., 2013, Nivala et al., 2008, Victorelli and Reis, 2020, Nielsen and Molich, 1990).

\section{Additional Checklist:}

- The system must provide all the tools available for navigation and manipulation of geographic data in an easy and organized way, allowing access and ease of use of the available functions.

- The user must be informed about the current default settings and how they can be changed.

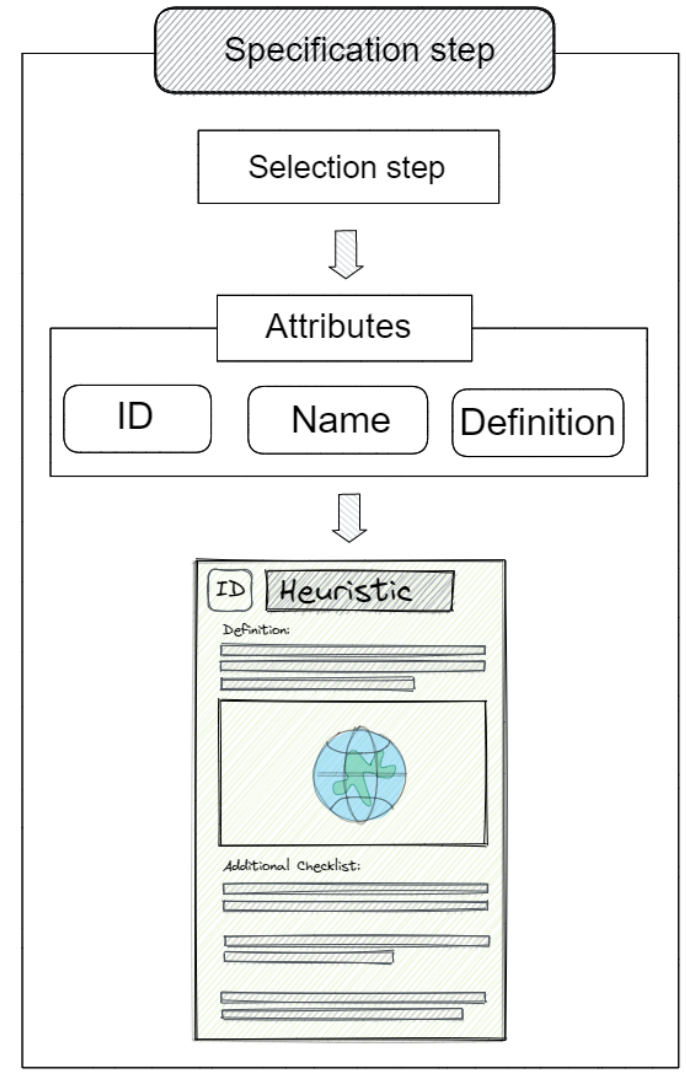

Figure 3. Representation of specification step.

- The map view must remain constantly visible while using the interface.

- The user must be able to control the application quickly and through known and commonly used essential functions.

- All information and tools must be provided in the main browser window, occupying an adequate part.

- Use short menu paths for the main functions or keep the main functions present at all times.

- Avoid visual clutter. The harmonious appearance, in general, should consist of clear contrast between visual elements, a balanced layout, and informative colors. Visual elements should guide users' gaze to the essential elements.

- There may be a modest number of advertisements and animations. However, these should not be placed to annoy the user.

- The application must provide users with information about errors and the possibility of their solution.

- The user should receive help using the tasks and other search functions. These aids can be presented as complementary information.

\subsection{Map status indication}

ID: $\mathrm{M} 2$

Category: Map view 
Definition: The map should only contain information relevant to the application, be intuitive and pleasant to use, and have accessible features and harmonic colors (Komarkova et al., 2011, 2007, Nivala et al., 2008).

\section{Additional Checklist:}

- Provide the user's geographic location referring to their position on the map.

- There must be a tool that centers the map around the user's geographic location.

- The answers provided to the user must be centered on the map and visualized differently from the other elements.

- The panoramic manipulation of the map must be available through manual drag and arrow keys.

- Map tools should be distinct but should not obscure too much information on the map.

- The map should be optimized for viewing on the device screen.

\subsection{Compatibility in representation levels}

ID: M3

\section{Category: Scale}

Definition: The map must be designed differently for distinct levels of visualization (Komarkova et al., 2011, Nivala et al., 2008, Tolochko, 2016).

\section{Additional Checklist:}

- Zoom buttons should be the most commonly visible elements of the map to allow users to change the scale.

- Allow only the zoom levels necessary for the map. Representative proportions or tractions should not be used.

- The scales must be presented in such a way that the size of the labels and toponyms are visible.

- Indicate to the user which zoom scale he is at and allow him to modify the scale on the bar itself.

- Set a minimum and maximum scale for display. Improper scaling or off-map access may confuse the user.

- Search results should be displayed on a scale where all information is visible, allowing the user to verify its veracity.

\subsection{Consistency and standardization of cartographic elements}

ID: M4

Category: Symbology

Definition: The symbols used must be used organically, following the primitive taxonomies applied to maps (Komarkova et al., 2007, Nivala et al., 2008, Roth, 2013).

\section{Additional Checklist:}

- Icons, navigation tools, and results should follow primitive and understandable taxonomies.

- The symbols presented as results should not cover the map too much or lie on top of each other.

\subsection{Accessible approach to the interactive environment}

ID: M5

Category: Interactivity

Definition: The interaction should be designed to support from the most basic to the most experienced user, ensuring the most diverse exploration objectives (Tolochko, 2016, Roth, 2013).

\section{Additional Checklist:}

- Allow the user can modify the results presented in the map by interacting with the legend.

\subsection{Assistance and recognition in search patterns}

ID: M6

Category: Research

Definition: Search engines must be presented in an intuitive way to the user (Kuparinen et al., 2013, Nivala et al., 2008).

\section{Additional Checklist:}

- The search feature must be a central function in the layout.

- Make clear to users the search criteria and how these criteria are related to the results.

- A list of previous searches should be saved and provided for quick replay.

- The search must support different types of searches.

- Indicate the reasons why the search was not found.

\subsection{Adaptability to different devices and browsers}

ID: M7

Category: Responsive design

Definition: The presentation of the map should not be interfered with in terms of device, screen, and browser settings (Komarkova et al., 2007, Kuparinen et al., 2013, Roth, 2013).

\section{Additional Checklist:}

- The interface must be scalable for different device screen sizes.

- The map must be visualized according to the properties of a device screen.

- The interface should work correctly in all web browsers.

\subsection{Name and structuring of cartographic metadata}

ID: M8

\section{Category: Metadata}

Definition: Geographic data must be made available in a structured way (Nivala et al., 2008, Tolochko, 2016). 


\section{Additional Checklist:}

- Information on geographic data must be provided, such as: (i) accuracy and validity of data; (ii) name(s) of the person(s) responsible; (iii) source of data; (iv) map projection; (v) others.

- Linked files or web pages (links) should be made available as complementary information.

\subsection{Consistency in the presentation of information and content}

ID: M9

Category: Additional information

Definition: The user must have easy access to complementary information. However, these information must not be superimposed places as the primary information presented on the map (Komarkova et al., 2007, Kuparinen et al., 2013, Nivala et al., 2008, Tolochko, 2016).

\section{Additional Checklist:}

- In the foreground, provide only the relevant information. As additional information such as: (i) embedded texts; (ii) links; (iii) images; (iv) graphics; (v) videos; (vi) metadata; (vii) user-generated content must be user-generated in the background, such as info windows, buttons, or map features.

- Additional software and plugins should not be needed.

- Provide the Uniform Resource Locator (URL) of the map apply.

\subsection{Permission to export maps and results obtained}

ID: M10

\section{Category: Data output}

Definition: The user must have access to functions that allow the export of the map(s) and the obtained result(s) (Komarkova et al., 2007).

\section{Additional Checklist:}

- Provide a function that allows printing the map(s) with the geographic information selected by the user.

\section{Next steps}

We will carry out the validation and refinement steps of the set of heuristics to complete our study. In the validation step, we will perform output 14 - Heuristic Evaluation (Figure 4) and output 16 - user tests.

Four groups composed of two experts will perform the Heuristic Assessment. These groups will be divided into two control groups (A and B), using Nielsen's heuristics, and two experimental groups ( $\mathrm{C}$ and $\mathrm{D})$, using our proposed heuristics. In addition, groups $\mathrm{A}$ and $\mathrm{C}$ will be composed of usability specialists, and groups $\mathrm{B}$ and $\mathrm{D}$ by cartographers.

The experts will verify the performance of the heuristics in identifying usability problems in the system, and the results will be analyzed in terms of five criteria (Quiñones et al., 2018), namely:

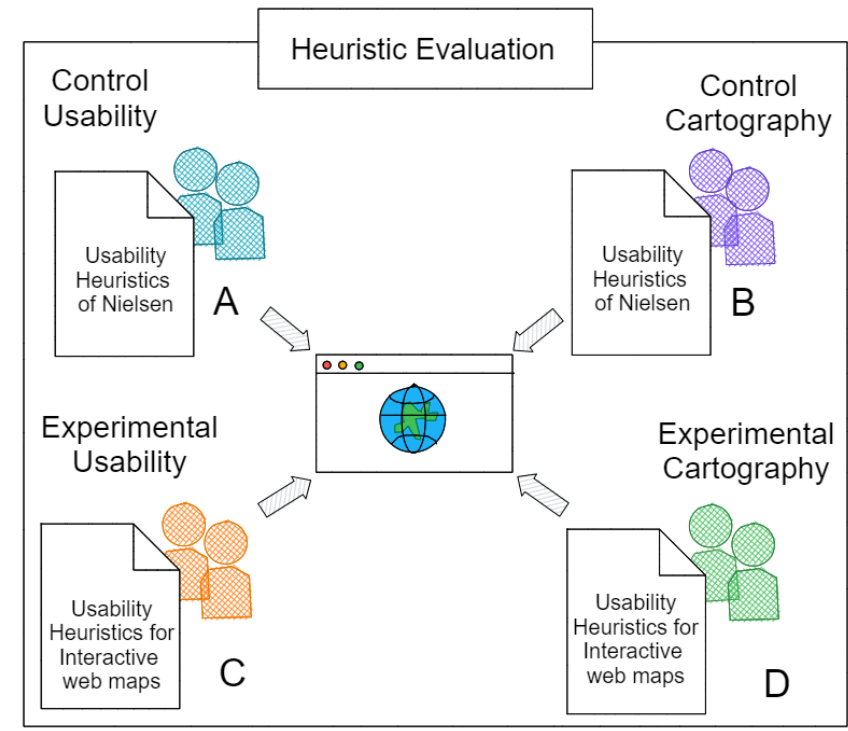

Figure 4. Heuristic Evaluation to be applied in the Validation step.

- $\mathrm{C} 1$ - numbers of correct and incorrect problem associations for heuristics;

- $\mathrm{C} 2$ - number of usability issues identified;

- C3 - Number of specific usability issues identified;

- C4 - Number of usability issues that qualify as more severe;

- C5 - Number of usability issues that qualify as most critical;

For output 16, users from different technical backgrounds will use the same system inspected in output 14 to verify if the usability issues identified in the Heuristic Evaluation are real problems ordinary users face.

Finally, a refined document will be produced, with details about the heuristics' transformation, refinement, or exclusion. This document should describe all modifications (why and how) and which steps should be repeated.

\section{Conclusion}

In this work, we proposed a guide to help develop and analyze the quality of interactive maps on the web to mitigate the existing gap of analyzing the user's interaction with the map. For this, we use the methodology proposed by Quiñones et al. (2018) to develop our domain-specific usability heuristics. This approach has a complete process involving steps ranging from identifying elements in the literature to validating the proposed set.

Among the proposed steps, we performed five (exploratory, descriptive, correlational, selection, and specification), reaching a set of ten usability heuristics for interactive maps on the web. In the next phase of our research, we will execute the validation and refinement steps. A heuristic evaluation will be applied with usability and cartographers experts and tests with users from different technical levels in the validation stage. 
During the entire process of creating the usability heuristics, we sought to integrate the cartographic concepts necessary for the application and geographic representation systems with the usability concepts to ensure better interaction between the user and the map. For this, two experts performed the correlational step, each one in a respective area: cartography and HCI. In the same way, we will perform the validation step.

Finally, we understand that the uniqueness of some interactive maps on the web can generate the need to create even more specific usability heuristics. Nevertheless, the proposed heuristics represent a new set for analyzing user interaction with interactive maps on the web.

\section{Acknowledgment}

This study was financed in part by the Coordenação de Aperfeiçoamento de Pessoal de Nível Superior - Brasil (CAPES) - Finance Code 001

\section{References}

Dykes, J., MacEachren, A. M. and Kraak, M.-J., 2005. Exploring geovisualization. Elsevier.

Griffin, A. L., Robinson, A. C. and Roth, R. E., 2017a. Envisioning the future of cartographic research.

Griffin, A. L., White, T., Fish, C., Tomio, B., Huang, H., Sluter, C. R., Bravo, J. V. M., Fabrikant, S. I., Bleisch, S., Yamada, M. et al., 2017b. Designing across map use contexts: A research agenda. International Journal of Cartography 3(sup1), pp. 90-114.

ISO, 1998. Ergonomic requirements for office work with visual display terminals (vdts) - part 11: Guidance on usability. Standard, International Organization for Standardization.

Komarkova, J., Sedlak, P., Novak, M., Musilova, A. and Slavikova, V., 2011. Methods of usability evaluation of web-based geographic information systems. International Journal of Systems Applications, Engineering \& Development 5(1), pp. 33-41.

Komarkova, J., Visek, O. and Novak, M., 2007. Heuristic evaluation of usability of geoweb sites. In: International Symposium on Web and Wireless Geographical Information Systems, Springer, pp. 264-278.

Kuparinen, L., Silvennoinen, J. and Isomäki, H., 2013. Introducing usability heuristics for mobile map applications.

Muehlenhaus, I., 2013. Web cartography: map design for interactive and mobile devices. CRC Press.

Nielsen, J. and Molich, R., 1990. Heuristic evaluation of user interfaces. In: Proceedings of the SIGCHI conference on Human factors in computing systems, pp. 249256.

Nivala, A.-M., Brewster, S. and Sarjakoski, T. L., 2008. Usability evaluation of web mapping sites. The Cartographic Journal 45(2), pp. 129-138.

Quiñones, D., Rusu, C. and Rusu, V., 2018. A methodology to develop usability/user experience heuristics. Computer standards \& interfaces 59, pp. 109-129.
Robinson, A. C., Demšar, U., Moore, A. B., Buckley, A., Jiang, B., Field, K., Kraak, M.-J., Camboim, S. P. and Sluter, C. R., 2017. Geospatial big data and cartography: research challenges and opportunities for making maps that matter. International Journal of Cartography 3(sup1), pp. 32-60.

Roth, R. E., 2013. Interactive maps: What we know and what we need to know. Journal of Spatial Information Science 2013(6), pp. 59-115.

Roth, R. E., Çöltekin, A., Delazari, L., Filho, H. F., Griffin, A., Hall, A., Korpi, J., Lokka, I., Mendonça, A., Ooms, K. et al., 2017. User studies in cartography: opportunities for empirical research on interactive maps and visualizations. International Journal of Cartography 3(sup1), pp. 61-89.

Rusu, C., Muñoz, R., Roncagliolo, S., Rudloff, S., Rusu, V. and Figueroa, A., 2011. Usability heuristics for virtual worlds. In: Proceedings of the Third International Conference on Advances in Future Internet, ser, Citeseer, pp. 16-19.

Sack, C., 2017. Web mapping. The Geographic Information Science and Technology Body of Knowledge.

Shekhar, S., Xiong, H. and Zhou, X., 2017. Encyclopedia of GIS. 2nd edn, Springer Publishing Company, Incorporated.

Solano, A., Rusu, C., Collazos, C., Roncagliolo, S., Arciniegas, J. L. and Rusu, V., 2011. Usability heuristics for interactive digital television. In: the third international conference on advances in future Internet, Citeseer, pp. 60-63.

Tolochko, R. C., 2016. Contemporary professional practices in interactive web map design. PhD thesis.

Vi, S., da Silva, T. S. and Maurer, F., 2019. User experience guidelines for designing hmd extended reality applications. In: IFIP Conference on Human-Computer Interaction, Springer, pp. 319-341.

Victorelli, E. Z. and Reis, J. C. D., 2020. Human-data interaction design guidelines for visualization systems. In: Proceedings of the 19th Brazilian Symposium on Human Factors in Computing Systems, pp. 1-10.

Vincent, K., Roth, R. E., Moore, S. A., Huang, Q., Lally, N., Sack, C. M., Nost, E. and Rosenfeld, H., 2019. Improving spatial decision making using interactive maps: an empirical study on interface complexity and decision complexity in the north american hazardous waste trade. Environment and Planning B: Urban Analytics and City Science 46(9), pp. 1706-1723.

White, T., 2017. Symbolization and the visual variables. The Geographic Information Science \& Technology Body of Knowledge, 2nd ed.; UCGIS: Ithaca, NY, USA. 